\title{
Broken promises
}

\author{
Intense public support for clinical research can be a mixed blessing - and the hunt for a vaccine against \\ AIDS offers an important lesson for many biomedical initiatives on what can go wrong.
}

ast year's failed clinical trial for Merck's HIV vaccine led many to claim that AIDS vaccine research is in crisis. And at a meeting

-last week in Bethesda, Maryland, to examine how the field should proceed, discussion centred on the need for a "mid-course correction", as Warner Greene, director of the Gladstone Institute of Virology and Immunology in San Francisco, put it.

The large-scale trial for Merck's candidate vaccine was welldesigned and had been launched with considerable optimism. Yet the vaccine not only failed to lower patients' viral loads, it actually boosted the risk of infection in some groups. And worse, there are few strikingly different vaccine candidates in the pipeline ready for human trials. The PAVE 100 trial for a vaccine candidate similar to Merck's has already been cut in size, and discussions are taking place on whether the trial should go ahead at all.

But if the AIDS vaccine field is in crisis, it is partly a crisis of its own making - one that holds lessons for many other areas of biomedical research. Decisions to move Merck's vaccine candidate and a previous failed candidate into clinical trials were based only partly on science. Also a factor was the field's need to show the public that progress is being made, thereby justifying the millions of dollars it receives from philanthropists and taxpayers. Historically, AIDS research has been afforded especially strong public support in the United States, but that means it works under an unusually intense public microscope. When promised results are not delivered, it's a double blow to the field, setting back the search for therapies while eroding public support.

This is an important lesson for other fields, most notably those, such as autism, Parkinson's and stem-cell research, that are funded by a new breed of philanthropic organizations and agencies that use a more 'business-like' management model. The end-point and milestone-driven funding model used by these organizations has become increasingly fashionable, as has their practice of setting more directed research priorities at the outset.

But there are consequences of the business-like approach that cannot be ignored. One is that trade-offs inevitably have to be made - often at the expense of basic research. The AIDS model provides a perfect example of this. When times are flush, as they were at the turn of this century, there is enough money to support clinical and basic researchers alike. When times become lean, as they are now, basic science loses out to the infrastructure that has been committed to clinical trials and 'big science' initiatives, such as the US\$300-million Center for HIV-AIDS Vaccine Immunology. The US National Institute of Allergy and Infectious Diseases, the leading player in the US AIDS initiative, is now spending slightly more of its extramural AIDS-vaccine budget on testing vaccines than on discovering new candidates. As a result, the development

"Researchers do not serve themselves well by promising results they cannot deliver." pipeline has run dry of new ideas. To the agency's credit, it recognizes the problem and its director, Anthony Fauci, has pledged to "turn the knob in the direction of discovery" (see page 516 ).

The other consequence of setting explicit milestones is the risk of public backlash when the results aren't delivered. AIDS vaccine research has made numerous promises it could not keep, such as when President Bill Clinton declared in 1997 that the nation would produce an AIDS vaccine within a decade. With every failure, public scrutiny grows. On $23 \mathrm{March}$, for instance, an article by the nonprofit AIDS Healthcare Foundation appeared in The Baltimore Sun calling for an end to clinical trials for AIDS vaccines.

Yet rather than learning from AIDS research, other fields are repeating its mistakes. The California Institute for Regenerative Medicine, for example, has promised to deliver "clinical proof-of-principle that transplanted cells derived from pluripotent cells can be used to restore function for at least one disease" by the end of its ten-year mandate. If this goal isn't met, the state's public, which voted to create and fund the institute, is likely to wonder whether its investment was worthwhile.

Researchers have no choice but to reach out to the public for support. But when they do so, they do not serve themselves well by promising results they cannot deliver.

\section{Double vision}

\section{The need to transform the world's energy technology is even greater than many perceive.}

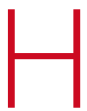
ow much energy, and of what sort, should we expect the world to be generating in the decades to come? This is a question of crucial importance to economics, development and the management of climate change. But as the Commentary on page 531 argues, it is not an easy question to answer (see also News on page 508).

In 2000, the Intergovernmental Panel on Climate Change introduced a range of 'emissions scenarios' that sought to estimate the amount of carbon emitted over the twenty-first century for a range of different assumptions. Climate modellers have since used a small subset of these scenarios as the basis for their analyses of possible climate change. But these scenarios are not without their controversies. One is their much-criticized approach to currency exchange rates, which some economists say overstates the impact of industrialization in developing countries. Another, it turns out, is not so much a problem with the scenarios themselves, but with the way some of them are seen as being 'business-as-usual' - in other words, what would happen if we do nothing differently.

That perception leads to an apparently straightforward measure of 